\title{
ON FILLING FAMILIES OF FINITE SUBSETS OF THE CANTOR SET
}

\author{
PANDELIS DODOS AND VASSILIS KANELLOPOULOS
}

\begin{abstract}
Let $\varepsilon>0$ and $\mathcal{F}$ be a family of finite subsets of the Cantor set $\mathcal{C}$. Following D. H. Fremlin, we say that $\mathcal{F}$ is $\varepsilon$-filling over $\mathcal{C}$ if $\mathcal{F}$ is hereditary and for every $F \subseteq \mathcal{C}$ finite there exists $G \subseteq F$ such that $G \in \mathcal{F}$ and $|G| \geq \varepsilon|F|$. We show that if $\mathcal{F}$ is $\varepsilon$-filling over $\mathcal{C}$ and $C$-measurable in $[\mathcal{C}]^{<\omega}$, then for every $P \subseteq \mathcal{C}$ perfect there exists $Q \subseteq P$ perfect with $[Q]^{<\omega} \subseteq \mathcal{F}$. A similar result for weaker versions of density is also obtained.
\end{abstract}

\section{INTRODUCTION}

Let $X$ be a set and $\varepsilon>0$. A family $\mathcal{F} \subseteq[X]^{<\omega}$ is said to be $\varepsilon$-filling over $X$ if $\mathcal{F}$ is hereditary (i.e. for every $F \in \mathcal{F}$ and every $G \subseteq F$ we have $G \in \mathcal{F}$ ) and for every $F \in[X]^{<\omega}$ there exists $G \subseteq F$ with $G \in \mathcal{F}$ and $|G| \geq \varepsilon|F|$. The notion of an $\varepsilon$-filling family is due to D. H. Fremlin [6], who posed the following problem. For which cardinals $\kappa, \lambda$ we have that whenever $|X|=\kappa$ and $\mathcal{F} \subseteq[X]^{<\omega}$ is $\varepsilon$-filling, then there exists $A \subseteq X$ with $|A|=\lambda$ and such that $[A]^{<\omega} \subseteq \mathcal{F}$ ? It is well-known that if $\kappa=\omega$, then $\lambda<\omega$. A classical example is the Schreier family $\mathcal{S}=\{F \subseteq \omega:|F| \leq \min F+1\}$. On the other hand, D. H. Fremlin has shown ([6], Corollary 6D) that large cardinal hypotheses imply the consistency of the statement that for every $\varepsilon$-filling family $\mathcal{F}$ over $\mathfrak{c}$, there exists $A \subseteq \mathfrak{c}$ infinite with $[A]^{<\omega} \subseteq \mathcal{F}$.

In this paper, we look at the problem when $X$ is the Cantor set $\mathcal{C}=2^{\omega}$. Notice that $[\mathcal{C}]^{<\omega}$ has the structure of a Polish space, being the direct sum of $[\mathcal{C}]^{k}(k \geq 1)$. S. A. Argyros, J. Lopez-Abad and S. Todorčević asked whether the above mentioned result of Fremlin is valid without extra set-theoretic assumptions provided that $\mathcal{F}$ is reasonably definable. We prove the following theorem which answers this question positively.

Theorem A. Let $\mathcal{F}$ be an $\varepsilon$-filling family over $\mathcal{C}$. If $\mathcal{F}$ is $C$-measurable in $[\mathcal{C}]^{<\omega}$, then for every $P \subseteq \mathcal{C}$ perfect there exists $Q \subseteq P$ perfect with $[Q]^{<\omega} \subseteq \mathcal{F}$.

Actually we prove a more general result (Theorem2 in the main text) which implies, for instance, that Theorem A is valid for an arbitrary $\varepsilon$-filling family in the Solovay Model.

\footnotetext{
${ }^{1} 2000$ Mathematics Subject Classification: 03E15, 05D10, 46B15.

${ }^{2}$ Research supported by a grant of EPEAEK program "Pythagoras".
} 
Our second result concerns weaker versions of density. For every $\mathcal{F} \subseteq[\mathcal{C}]^{<\omega}$ and every $n \geq 1$ let $d_{\mathcal{F}}(n)$ be the density of $\mathcal{F}$ at $n$, that is

$$
d_{\mathcal{F}}(n)=\min _{F \in[\mathcal{C}]^{n}} \max \{|G|: G \subseteq F \text { and } G \in \mathcal{F}\} .
$$

Notice that $\mathcal{F}$ is $\varepsilon$-filling if and only if $\mathcal{F}$ is hereditary and $\frac{d_{\mathcal{F}}(n)}{n} \geq \varepsilon$ for all $n \geq 1$. Although every $C$-measurable $\varepsilon$-filling family $\mathcal{F}$ over $\mathcal{C}$ is not compact, Fremlin has shown that for every $f: \omega \rightarrow \omega$ with $n \geq f(n)>0$ for all $n \geq 1$ and $\lim \frac{f(n)}{n}=0$ there exists a compact and hereditary family $\mathcal{F}$, closed in $[\mathcal{C}]^{<\omega}$ and such that $d_{\mathcal{F}}(n) \geq f(n)$ for every $n \geq 1$ (see [6], Proposition 4B). The following theorem shows, however, that any such family $\mathcal{F}$ must still be large.

Theorem B. Let $\mathcal{F} \subseteq[\mathcal{C}]^{<\omega}$ hereditary. Assume that $\mathcal{F}$ has the Baire property in $[\mathcal{C}]<\omega$ and satisfies

$$
\text { (*) } \quad \lim \sup \frac{\log _{2} d_{\mathcal{F}}\left(2^{n}\right)}{\log _{2} n}=+\infty .
$$

Then for every $k \geq 1$ there exists $P \subseteq \mathcal{C}$ perfect such that $[P]^{k} \subseteq \mathcal{F}$.

The proof of Theorem B is based on A. Blass' theorem 44. Theorem B has the following consequence which shows that we can increase the density of $\mathcal{F}$ by passing to a perfect subset. In particular, if $\mathcal{F}$ is $C$-measurable and satisfies equation (*) above, then for every $f: \omega \rightarrow \omega$ with $n \geq f(n)>0$ for all $n \geq 1$ and $\lim \frac{f(n)}{n}=0$ and every perfect subset $P$ of $\mathcal{C}$ there exists $Q \subseteq P$ perfect such that the density of $\mathcal{F}$ in $Q$ is greater or equal to $f$. We also include some connections of the above results with Banach spaces.

Acknowledgments. We would like to thank Professor Spiros A. Argyros for bringing the problem to our attention as well as for suggesting Corollary 18 and the Banach space theoretic implications. We also thank Alexander D. Arvanitakis for many stimulating conversations.

\section{Preliminaries}

We let $\omega=\{0,1, \ldots\}$. The cardinality of a set $A$ is denoted by $|A|$. By $<$ we denote the (strict) lexicographical ordering on the Cantor set $\mathcal{C}=2^{\omega}$. If $A, B \subseteq \mathcal{C}$, then we write $A<B$ if for every $x \in A$ and every $y \in B$, we have $x<y$. For every $n \geq 1$ and every $P \subseteq \mathcal{C}$, by $[P]^{n}$ we denote the set of all $<$-increasing sequences of $P$ of cardinality $n$, while by $[P]^{<\omega}$ the set of all finite $<$-increasing sequences of $P$.

By $2^{<\omega}$ we denote the Cantor tree, i.e. the set of all finite sequences of 0 's and 1 's, equipped with the (strict) partial ordering $\sqsubset$ of initial segment. If $s, t \in 2^{<\omega}$, then by $s^{\frown} t$ we denote their concatenation. For every $s \in 2^{<\omega}$, the length $\ell(s)$ of $s$ is defined to be the cardinality of the set $\left\{t \in 2^{<\omega}: t \sqsubset s\right\}$. For every $n \in \omega$, by $2^{n}$ we denote the set of all sequences in $2^{<\omega}$ of length $n$, while for every $n \geq 1$ by $2^{<n}$ we denote the set of all sequences of length less than $n$. For every $s, t \in 2^{<\omega}$ 
we denote by $s \wedge t$ the $\sqsubset$-maximal node $w$ such that $w \sqsubseteq s$ and $w \sqsubseteq t$. Similarly, if $x, y \in \mathcal{C}$, then by $x \wedge y$ we denote the $\sqsubset$-maximal node $t$ of $2^{<\omega}$ with $t \sqsubset x$ and $t \sqsubset y$. We write $s \prec t$ if $w^{\frown} 0 \sqsubseteq s$ and $w^{\urcorner} 1 \sqsubseteq t$, where $w=s \wedge t$.

We view every subset of $2^{<\omega}$ as a subtree of $2^{<\omega}$ equipped with the induced partial ordering. For every $m \in \omega$ and every subtree $T$ of $2^{<\omega}$ by $T(m)$ we denote the $m$-level of $T$, that is the set of all $t \in T$ such that $|\{s \in T: s \sqsubset t\}|=m$. A node $t \in T$ is said to be a splitting node of $T$ if $t$ has at least two immediate successors in $T$. By $\operatorname{Spl}(T)$ we denote the set of splitting nodes of $T$.

A subtree $T$ of $2^{<\omega}$ is said to be downwards closed if for every $t \in T$ the set $\{s: s \sqsubseteq t\}$ is a subset of $T$. Notice that if $T$ is a downwards closed subtree and $m \in \omega$, then $T(m)=\left\{t \in T: t \in 2^{m}\right\}$. The body [T] of $T$ is the set $\{x \in \mathcal{C}: x \mid n \in T \forall n \in \omega\}$, where $x \mid n=\left(x_{0}, \ldots, x_{n-1}\right) \in 2^{<\omega}$ if $n \geq 1$ and $x \mid 0=(\varnothing)$ if $n=0$. If $t \in T$, then we set $[T]_{t}=\{x \in[T]: t \sqsubset x\}$. In particular, for every $t \in 2^{<\omega}$ we have $\mathcal{C}_{t}=\{x \in \mathcal{C}: t \sqsubset x\}$.

If $A \subseteq 2^{<\omega}$, then the downwards closure $\hat{A}$ of $A$ is the set $\left\{s \in 2^{<\omega}: \exists t \in\right.$ $A$ with $s \sqsubseteq t\}$. Moreover, for every $F \subseteq \mathcal{C}$ we let $T_{F}=\{x \mid n: x \in F, n \in \omega\}$. Observe that $F$ is closed if and only if $F=\left[T_{F}\right]$. It is easy to see that if $F$ is a finite subset of $\mathcal{C}$, then $\left|\operatorname{Spl}\left(T_{F}\right)\right|=|F|-1$. Similarly if $A$ is a finite antichain of $2^{<\omega}$, then $|\operatorname{Spl}(\hat{A})|=|A|-1$.

A subtree $T$ of $2^{<\omega}$ is said to be pruned if for every $t \in T$ there exists $s \in T$ with $t \sqsubset s$. It is said to be skew if for every $m \in \omega$ we have $|T(m) \cap \operatorname{Spl}(T)| \leq 1$.

Let us recall the notion of the type $\tau$ of a downwards closed, pruned, skew subtree $T$ of $2^{<\omega}$ taken from Louveau-Shelah-Veličković [8]. We will only treat trees $T$ with $[T]$ finite. So, let $k \geq 2$ and let $T$ be a downwards closed, pruned, skew subtree of $2^{<\omega}$ such that $[T]$ has $k$ elements. The type of $T$ is a function $\tau:\{1, \ldots, k-1\} \rightarrow \omega$, where $\tau(n)$ is defined as follows. For every $n \in\{1, \ldots, k-1\}$ let $m \in \omega$ be the least such that $T(m)$ has $n+1$ nodes. Let $T(m-1)=\left\{s_{0} \prec \ldots \prec s_{n-1}\right\}$. Then $\tau(n)=d$, if $s_{d}$ is the unique splitting node of $T(m-1)$. Every type of a tree $T$ with $[T]=k$ will be called a $k$-type. It is easy to see that for every $k \geq 2$ there exist $(k-1)$ ! $k$-types. We remark that the above definition is equivalent to the initial one, given by A. Blass [4. If $F$ is a finite subset of $\mathcal{C}$, then we say that $F$ is of type $\tau$ if $T_{F}$ is skew and of type $\tau$. If $P \subseteq \mathcal{C}$ and $\tau$ is a $k$-type, then by $[P]_{\tau}^{k}$ we denote the set of all subsets of $P$ of type $\tau$.

We will also treat the following class of subtrees of $2^{<\omega}$ which are not downwards closed. A subtree $T$ of $2^{<\omega}$ is said to be regular dyadic if $T$ can be written in the form $T=\left(t_{s}\right)_{s \in 2^{<\omega}}$ such that for all $s_{1}, s_{2} \in 2^{<\omega}$ the following are satisfied.

(1) $s_{1} \sqsubset s_{2}$ (respectively $s_{1} \prec s_{2}$ ) if and only if $t_{s_{1}} \sqsubset t_{s_{2}}$ (respectively $t_{s_{1}} \prec t_{s_{2}}$ ).

(2) $\ell\left(s_{1}\right)=\ell\left(s_{2}\right)$ if and only if $\ell\left(t_{s_{1}}\right)=\ell\left(t_{s_{2}}\right)$.

It is easy to see that the representation of $T$ as $\left(t_{s}\right)_{s \in 2<\omega}$ is unique. In what follows when we deal with a regular dyadic subtree $T$ we will always use this unique 
representation. We also notice that if $T$ is a regular dyadic tree, then $[\hat{T}]$ is a perfect subset of $\mathcal{C}$ homeomorphic to $\mathcal{C}$.

Finally, we recall that a subset $A$ of an uncountable Polish space $X$ is $C$ measurable if it belongs to the smallest $\sigma$-algebra which is closed under the Souslin operation and contains the open sets. We remark that the class of $C$-measurable sets is strictly bigger than the $\sigma$-algebra generated by the analytic sets (see [7]).

\section{Definable $\varepsilon$-Filling Families}

We start with the following definition.

Definition 1. Let $\mathcal{F} \subseteq[\mathcal{C}]^{<\omega}$. The family $\mathcal{F}$ is said to have the Galvin property if for every $n \in \omega$ and every $P_{0}<\ldots<P_{n}$ perfect subsets of $\mathcal{C}$, there exist $Q_{0}, \ldots, Q_{n}$ such that the following hold.

(1) For all $i=0, \ldots, n, Q_{i}$ is a perfect subset of $P_{i}$.

(2) Either $Q_{0} \times \ldots \times Q_{n} \subseteq \mathcal{F}$ or $\left(Q_{0} \times \ldots \times Q_{n}\right) \cap \mathcal{F}=\varnothing$.

We notice that if for every $n \in \omega$ and every $P_{0}<\ldots<P_{n}$ perfect subsets of $\mathcal{C}$ the set $\mathcal{F} \cap\left(P_{0} \times \ldots \times P_{n}\right)$ has the Baire property in $P_{0} \times \ldots \times P_{n}$, then the family $\mathcal{F}$ has the Galvin property. This is a consequence of a theorem of F. Galvin (see [7, Theorem 19.6). Under the above terminology we have the following.

Theorem 2. Let $\varepsilon>0$ and $\mathcal{F}$ be an $\varepsilon$-filling family over $\mathcal{C}$. If $\mathcal{F}$ has the Galvin property, then for every perfect subset $P$ of $\mathcal{C}$ there exists $Q \subseteq P$ perfect such that $[Q]^{<\omega} \subseteq \mathcal{F}$.

For the proof of Theorem 2 we need the following definition.

Definition 3. Let $\mathcal{F} \subseteq[\mathcal{C}]^{<\omega}$ and $T=\left(t_{s}\right)_{s \in 2^{<\omega}}$ be a regular dyadic subtree of $2^{<\omega}$. We say that the tree $T$ decides for $\mathcal{F}$ if for every $n \in \omega$, every $0 \leq d \leq 2^{n}-1$ and every $F=\left\{s_{0} \prec \ldots \prec s_{d}\right\} \subseteq 2^{n}$ we have that the product $[\hat{T}]_{t_{s_{0}}} \times \ldots \times[\hat{T}]_{t_{s_{d}}}$ either is included in or is disjoint from $\mathcal{F}$. In the case where $[\hat{T}]_{t_{s_{0}}} \times \ldots \times[\hat{T}]_{t_{s_{d}}}$ is included in $\mathcal{F}$, then we say that $F$ is trapped in $\mathcal{F}$.

The following lemma is the combinatorial part of the proof of Theorem 2 .

Lemma 4. Let $\mathcal{F} \subseteq[\mathcal{C}]^{<\omega}$ with the Galvin property and $P$ be a perfect subset of $\mathcal{C}$. Then there exists a regular dyadic tree $T=\left(t_{s}\right)_{s \in 2}<\omega$ that decides for $\mathcal{F}$ and $[\hat{T}] \subseteq P$.

Proof. By recursion on the length of $s \in 2^{<\omega}$ we will build a regular dyadic tree $T=\left(t_{s}\right)_{s \in 2<\omega}$ and a family $\left(P^{s}\right)_{s \in 2<\omega}$ of subsets of $\mathcal{C}$ such that for all $n \in \omega$ the following are satisfied.

(1) For all $s \in 2^{n}, P^{s}$ is a perfect subset of $P$.

(2) If $n \geq 1$, then for all $s \in 2^{n-1}$ and $i \in\{0,1\}, P^{s^{\wedge} i} \subseteq P^{s} \cap \mathcal{C}_{t_{s^{\wedge}}}$. 
(3) For every $0 \leq d \leq 2^{n}-1$ and every $\left\{s_{0} \prec \ldots \prec s_{d}\right\} \subseteq 2^{n}$ we have that $P^{s_{0}} \times \ldots \times P^{s_{d}}$ either is included in or is disjoint from $\mathcal{F}$.

We start the construction. For $n=0$, we set $t_{\varnothing}=\varnothing$. By the Galvin property of $\mathcal{F}$, there exists $P^{\varnothing} \subseteq P$ perfect such that either $\left[P^{\varnothing}\right]^{1} \subseteq \mathcal{F}$ or $\left[P^{\varnothing}\right]^{1} \cap \mathcal{F}=\varnothing$. Then (1) and (3) are satisfied. Now assume that for some $n \in \omega,\left(t_{s}\right)_{s \in 2^{n}}$ and $\left(P^{s}\right)_{s \in 2^{n}}$ have been constructed. As the family $\left\{P^{s}: s \in 2^{n}\right\}$ consists of perfect subsets of $P$ and $P^{s} \subseteq \mathcal{C}_{t_{s}}$, we may select a sequence $\left(t_{s}\right)_{s \in 2^{n+1}}$ such that the following are satisfied.

(i) For all $s_{1}, s_{2} \in 2^{n+1}, \ell\left(t_{s_{1}}\right)=\ell\left(t_{s_{2}}\right)$.

(ii) For every $s \in 2^{n}$, the nodes $t_{s \frown 0}$ and $t_{s \frown 1}$ are successors of $t_{s}$ and $t_{s^{\frown} 0} \prec$ $t_{s\urcorner 1}$.

(iii) For all $s \in 2^{n}$ and $i \in\{0,1\}$, setting $Q^{s^{\wedge} i}=P^{s} \cap \mathcal{C}_{t_{s} \rightarrow i}$ we have that $Q^{s^{\frown} i}$ is a perfect subset of $P^{s}$.

Using the fact that $\mathcal{F}$ has the Galvin property, by an exhaustion argument over all subsets of $2^{n+1}$, we find for all $s \in 2^{n+1}$ a perfect set $P^{s} \subseteq Q^{s}$ such that condition (3) is satisfied. This completes the recursive construction.

We will check that $T=\left(t_{s}\right)_{s \in 2}<\omega$ satisfies all the desired properties. First we observe that $[\hat{T}] \subseteq P$ is an immediate consequence of (1), (2) and the fact that $P^{\varnothing} \subseteq P$. By (2) we also have that $[\hat{T}]_{t_{s}} \subseteq P^{s}$ for all $s \in 2^{<\omega}$. Hence, by (3) we get that $T$ decides for $\mathcal{F}$, as desired.

Lemma 5. Let $\mathcal{F} \subseteq[\mathcal{C}]^{<\omega}$ and $T=\left(t_{s}\right)_{s \in 2<\omega}$ a regular dyadic tree that decides for $\mathcal{F}$. Assume that $\mathcal{F}$ is $\varepsilon$-filling for some $\varepsilon>0$. Then the following hold.

(1) For every $n \in \omega$, there exists $F_{n} \subseteq 2^{n}$ with $\left|F_{n}\right| \geq \varepsilon \cdot 2^{n}$ and such that $F_{n}$ is trapped in $\mathcal{F}$.

(2) Let $n, k \in \omega$ with $k \leq n, F \subseteq 2^{n}$ and $G \subseteq 2^{k}$ such that $G$ is dominated by $F$ (i.e. for every $w \in G$ there exists $s \in F$ with $w \sqsubseteq s$ ). If $F$ is trapped in $\mathcal{F}$, then so does $G$.

Proof. (1) For every $s \in 2^{n}$, pick $x_{s} \in[\hat{T}]_{t_{s}}$. As $\mathcal{F}$ is $\varepsilon$-filling, there exists $F_{n}=$ $\left\{s_{0} \prec \ldots \prec s_{d-1}\right\} \subseteq 2^{n}$ with $d \geq \varepsilon \cdot 2^{n}$ and such that $\left\{x_{s}: s \in F_{n}\right\} \subseteq \mathcal{F}$. It follows that $\left([\hat{T}]_{t_{s_{0}}} \times \ldots \times[\hat{T}]_{t_{s_{d-1}}}\right) \cap \mathcal{F} \neq \varnothing$. Since the tree $T$ decides for $\mathcal{F}$, we conclude that $F_{n}$ is trapped in $\mathcal{F}$.

(2) First we notice that if $F$ is trapped in $\mathcal{F}$, then every subset of $F$ is also trapped in $\mathcal{F}$, as $\mathcal{F}$ is hereditary. Now let $G$ be dominated by $F$. There exists $F^{\prime}$ subset of $F$ with $\left|F^{\prime}\right|=|G|$ and such that for every $w \in G$ there exists a unique $s \in F^{\prime}$ with $w \sqsubseteq s$. Arguing as in (1) above we get that $G$ is trapped in $\mathcal{F}$, as desired.

For every regular dyadic tree $T=\left(t_{s}\right)_{s \in 2<\omega}$ we define a canonical Borel probability measure $\mu_{T}$ on $[\hat{T}]$ by assigning to every $[\hat{T}]_{t_{s}}$, with $s \in 2^{n}$ and $n \in \omega$, measure equal to $\frac{1}{2^{n}}$. That is, $\mu_{T}$ is the image of the usual measure on $\mathcal{C}$ induced by the natural homeomorphism between $\mathcal{C}$ and $[\hat{T}]$. We remark that $\mu_{T}$ is continuous (i.e. 
it vanishes on singletons) and regular. The final lemma consists of the analytic part of the argument.

Lemma 6. Let $\mathcal{F} \subseteq[\mathcal{C}]^{<\omega}$ and $T=\left(t_{s}\right)_{s \in 2<\omega}$ a regular dyadic tree that decides for $\mathcal{F}$. Assume that $\mathcal{F}$ is $\varepsilon$-filling for some $\varepsilon>0$. Then there exists $K \subseteq[\hat{T}]$ closed such that $\mu_{T}(K) \geq \varepsilon$ and $[K]^{<\omega} \subseteq \mathcal{F}$.

Proof. By Lemma $5(1)$, for every $n \in \omega$ there exists $F_{n} \subseteq 2^{n}$ with $\left|F_{n}\right| \geq \varepsilon \cdot 2^{n}$ and such that $F_{n}$ is trapped in $\mathcal{F}$. Define

$$
C_{n}=\bigcup_{s \in F_{n}}[\hat{T}]_{t_{s}}
$$

Then $C_{n}$ is a clopen subset of $[\hat{T}]$ and moreover $\mu_{T}\left(C_{n}\right) \geq \varepsilon$ for every $n \in \omega$. Let us denote by $\mathcal{K}([\hat{T}])$ the hyperspace of all compact subsets of $[\hat{T}]$ equipped with the Vietoris topology. It is a compact metrizable space (see [7]). Hence, there exist an infinite subset $L$ of $\omega$ and $K \in \mathcal{K}([\hat{T}])$ such that the sequence $\left(C_{n}\right)_{n \in L}$ is convergent to $K$. As the measure $\mu_{T}$ is regular, the map $\mathcal{K}([\hat{T}]) \ni K \mapsto \mu_{T}(K)$ is upper semicontinuous. It follows that

$$
\mu_{T}(K) \geq \limsup _{n \in L} \mu_{T}\left(C_{n}\right) \geq \varepsilon .
$$

It remains to show that $[K]^{<\omega} \subseteq \mathcal{F}$. Indeed, let $\left\{x_{0}<\ldots<x_{l}\right\} \subseteq K$. Since $K \subseteq[\hat{T}]$, there exist $k \in \omega$ and $\left\{w_{0} \prec \ldots \prec w_{l}\right\} \subseteq 2^{k}$ such that $t_{w_{i}} \sqsubset x_{i}$ for all $i=0, \ldots, l$ (notice that $\left.\ell\left(t_{w_{0}}\right)=\ldots=\ell\left(t_{w_{l}}\right)\right)$. The sequence $\left(C_{n}\right)_{n \in L}$ converges to $K$ and so there exists $n_{0} \in L$ such that for all $n \in L$ with $n \geq n_{0}$, the set $\left\{t_{s}: s \in F_{n}\right\}$ dominates the set $\left\{t_{w_{0}}, \ldots, t_{w_{l}}\right\}$. The tree $T$ is regular dyadic and so $F_{n}$ dominates $\left\{w_{0}, \ldots, w_{l}\right\}$. As every $F_{n}$ is trapped in $\mathcal{F}$, by Lemma 5 (2) we get that $\left\{w_{0}, \ldots, w_{l}\right\}$ is trapped in $\mathcal{F}$ too. This clearly implies that $\left\{x_{0}, \ldots, x_{l}\right\} \in \mathcal{F}$ and the proof is completed.

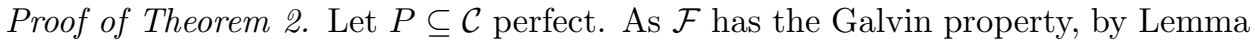
4 there exists a regular dyadic tree $T$ such that $T$ decides for $\mathcal{F}$ and $[\hat{T}] \subseteq P$. Since $\mathcal{F}$ is $\varepsilon$-filling, by Lemma 6 there exists $K \subseteq[\hat{T}]$ closed with $\mu_{T}(K) \geq \varepsilon$ and such that $[K]^{<\omega} \subseteq \mathcal{F}$. As $\mu_{T}$ is continuous, $K$ is an uncountable closed subset of $P$ and the result follows.

Consequences. We notice that for every Polish space $X$, every closed subset $F$ of $X$ and every $C$-measurable subset $A$ of $X$, the set $A \cap F$ is $C$-measurable in $F$. Invoking the classical fact that every $C$-measurable subset of a Polish space has the Baire property (hence, by the remarks at the beginning of the section, the Galvin property too), we get the following corollary of Theorem 2 .

Corollary 7. Let $\mathcal{F} \subseteq[\mathcal{C}]^{<\omega}$ be $\varepsilon$-filling. If $\mathcal{F}$ is $C$-measurable in $[\mathcal{C}]^{<\omega}$, then for every $P \subseteq \mathcal{C}$ perfect, there exists $Q \subseteq P$ perfect with $[Q]^{<\omega} \subseteq \mathcal{F}$. 
As Projective Determinacy (PD) implies that every projective set in a Polish space has the Baire property (see [7, Theorem 38.17), under PD, Corollary 7 is also true for every projective set.

There are some natural limitations on the possibility of extending Corollary 7 for an arbitrary $\varepsilon$-filling family. Indeed, let $B$ be a Bernstein set, that is a subset of $\mathcal{C}$ such that neither $B$ nor $\mathcal{C} \backslash B$ contain a perfect set. Setting $\mathcal{F}=[B]^{<\omega} \cup[\mathcal{C} \backslash B]^{<\omega}$ we see that $\mathcal{F}$ is $1 / 2$-filling, yet there does not exist a perfect set $P$ with $[P]^{<\omega} \subseteq \mathcal{F}$. Notice however that the above counterexample is depended on the Axiom of Choice. As a matter of fact, every counterexample known to us depends on the Axiom of Choice. This is not an accident. As in the proof of Theorem 2 we made no use of the Axiom of Choice, we have the following corollary.

Corollary 8. Assume $Z F+D C$ and the statement that "every subset of a Polish space has the Baire property". Then for every $\varepsilon$-filling family $\mathcal{F}$ over $\mathcal{C}$ and every $P \subseteq \mathcal{C}$ perfect, there exists $Q \subseteq P$ perfect such that $[Q]^{<\omega} \subseteq \mathcal{F}$.

We notice that the hypotheses of Corollary 8 hold in the Solovay Model [9] (see also [3] section 5.3, for a discussion about this in a different but related context). A similar result has been also obtained by A. W. Apter and M. Džamonja [1].

Remark 1. It follows by Corollary 7 , that if $\mathcal{F} \subseteq[\mathcal{C}]^{<\omega}$ is analytic and $\varepsilon$-filling, then $\mathcal{F}$ cannot be compact; that is there exists $A \subseteq \mathcal{C}$ infinite such that $[A]^{<\omega} \subseteq \mathcal{F}$. We should point out that this can also be derived by the results of D. H. Fremlin in [6. To see this, one argues by contradiction. So, assume that $\mathcal{F} \subseteq[\mathcal{C}]^{<\omega}$ is analytic, compact and $\varepsilon$-filling for some $\varepsilon>0$. It was observed by S. A. Argyros, J. Lopez-Abad and S. Todorčević that the rank of $\mathcal{F}$ is a countable ordinal whenever $\mathcal{F}$ is analytic and compact. This follows by a standard application of the KunenMartin theorem (see [7, Theorem 31.1). By Lemma 2C in [6] applied to the ideal $\mathcal{I}$ of countable subsets of $\mathcal{C}$, we get that the rank of $\mathcal{F}$ must be greater or equal to $\omega_{1}$, which is a contradiction.

Remark 2. By modifying the proof of Theorem 2 we have the following result for an arbitrary family $\mathcal{F}$.

Theorem 9. Let $\varepsilon>0$ and $\mathcal{F} \subseteq[\mathcal{C}]^{<\omega}$ be an arbitrary $\varepsilon$-filling family. Then for every $P \subseteq \mathcal{C}$ perfect there exists $Q \subseteq P$ perfect such that for every $R \subseteq Q$ perfect and every $k \geq 1$ the set $\mathcal{F} \cap[R]^{k}$ is dense in $[R]^{k}$.

The proof of Theorem 9 follows the arguments of the proof of Theorem 2. The only severe change is that of the notion of a regular dyadic the decides for $\mathcal{F}$. Specifically, Definition 3 is modified as follows.

Definition 10. Let $\mathcal{F} \subseteq[\mathcal{C}]^{<\omega}$ and $T=\left(t_{s}\right)_{s \in 2<\omega}$ be a regular dyadic subtree of $2^{<\omega}$. We say that the tree $T$ weakly decides for $\mathcal{F}$ if for every $n \in \omega$, every $0 \leq d \leq 2^{n}-1$ and every $F=\left\{s_{0} \prec \ldots \prec s_{d}\right\} \subseteq 2^{n}$ we have that one of the the following (mutually exclusive) alternatives holds. 
(1) Either $\left([\hat{T}]_{t_{s_{0}}} \times \ldots \times[\hat{T}]_{t_{s_{d}}}\right) \cap \mathcal{F}=\varnothing$, or

(2) for every $i=0, \ldots, d$ and every $Q_{i} \subseteq[\hat{T}]_{t_{s_{i}}}$ perfect we have $\left(Q_{0} \times \ldots \times Q_{d}\right) \cap$ $\mathcal{F} \neq \varnothing$.

In the case where alternative (2) holds, then we say that $F$ is weakly trapped in $\mathcal{F}$.

It can be easily checked that the arguments of the proofs of Lemmas 4,5 and 6 can be carried out using the above definitions, yielding the proof of Theorem 9 ,

\section{FAMilies OF WEAKer DENSITY}

This section is devoted to the proof of Theorem B stated in the introduction. For the convenience of the reader, let us present the example of Fremlin which provides closed hereditary families over $\mathcal{C}$ (of weaker density) for which Theorem 2 is not valid.

Example 1. Let $f: \omega \rightarrow \omega$ be any function such that $n \geq f(n)>0$ for all $n \geq 1$ and $\lim \frac{f(n)}{n}=0$. Then there exists a family $\mathcal{F} \subseteq[\mathcal{C}]^{<\omega}$ such that the following hold.

(1) $\mathcal{F}$ is closed in $[\mathcal{C}]^{<\omega}$ and hereditary.

(2) $d_{\mathcal{F}}(n) \geq f(n)$ for all $n \geq 1$.

(3) There does not exist $A \subseteq \mathcal{C}$ infinite with $[A]^{<\omega} \subseteq \mathcal{F}$.

Indeed, we can chose a strictly increasing sequence $\left(n_{k}\right)_{k \in \omega}$ such that $n_{0}=1$ and $\sup _{i \geq n_{k}} \frac{f(i)}{i} \leq \frac{1}{2^{k}}$ for all $k \geq 1$. We set

$$
\mathcal{F}=\bigcup_{k \in \omega} \bigcup_{t \in 2^{k}}\left\{G: G \subseteq \mathcal{C}_{t} \text { and }|G| \leq\left\lceil n_{k+1} / 2^{k}\right\rceil\right\} .
$$

It is easy to see that (1) and (3) are satisfied. To verify (2), let $F \subseteq \mathcal{C}$ with $|F|=n$. Let $k \in \omega$ be such that $n_{k} \leq n<n_{k+1}$. Then $F$ is partitioned in $\left\{F \cap \mathcal{C}_{t}\right\}_{t \in 2^{k}}$. There exists $t_{0} \in 2^{k}$ such that $\left|F \cap \mathcal{C}_{t_{0}}\right| \geq\left\lceil n / 2^{k}\right\rceil$. Let $G$ be any subset of $F \cap \mathcal{C}_{t_{0}}$ with $|G|=\left\lceil n / 2^{k}\right\rceil$. By the definition of $\mathcal{F}$ and the fact that $n<n_{k+1}$, we see that $G \in \mathcal{F}$. As $n \geq n_{k}$, we have $\frac{f(n)}{n} \leq \frac{1}{2^{k}}$ and so $f(n) \leq\left\lceil n / 2^{k}\right\rceil \leq d_{\mathcal{F}}(n)$.

Let us pass now to the proof of the main result of this section (Theorem 16 below). Observe that for every $P \subseteq \mathcal{C}$ perfect and every $k$-type $\tau$, the set $[P]_{\tau}^{k}$ is non-empty. We will need a finite version of this fact. To this end, we make the following definitions.

Definition 11. Let $n \geq 1$. A finite subtree $T$ of the Cantor tree $2^{<\omega}$ is said to be $n$-increasing if $T$ can be written in the form $T=\left(t_{s}\right)_{s \in 2<n}$ such that for all $s_{1}, s_{2} \in 2^{<n}$ the following are satisfied.

(1) $t_{s_{1}} \sqsubset t_{s_{2}}$ (respectively $t_{s_{1}} \prec t_{s_{2}}$ ) if and only if $s_{1} \sqsubset s_{2}$ (respectively $s_{1} \prec s_{2}$ ).

(2) If $\ell\left(s_{1}\right)=\ell\left(s_{2}\right)$ and $s_{1} \prec s_{2}$, then $\ell\left(t_{s_{1}}\right)<\ell\left(t_{s_{2}}\right)$.

(3) If $\ell\left(s_{1}\right)<\ell\left(s_{2}\right)$, then $\ell\left(t_{s_{1}}\right)<\ell\left(t_{s_{2}}\right)$. 
Definition 12. A subset $F \subseteq \mathcal{C}$ with $|F|=2^{n}$ is said to be $2^{n}$-increasing if the set $\operatorname{Spl}\left(T_{F}\right)$ of splitting nodes of $T_{F}$ forms an n-increasing subtree of $2^{<\omega}$. The set of all $2^{n}$-increasing subsets of $\mathcal{C}$ will be denoted by $[\mathcal{C}]_{\Delta}^{2^{n}}$.

It is easy to see that if $F$ is a $2^{n}$-increasing subset of $\mathcal{C}$, then $T_{F}$ is a skew subtree of $2^{<\omega}$. The class of increasing subsets of $\mathcal{C}$ has the following stability property.

Lemma 13. Let $n \geq 2$ and $k \geq 1$ be such that $2^{n} \geq n^{k}$. Then for every $F \in[\mathcal{C}]_{\Delta}^{2^{n}}$ and every $G \subseteq F$ with $|G| \geq n^{k}$ there exists $H \subseteq G$ with $H \in[\mathcal{C}]_{\Delta}^{2^{k}}$.

Proof. Let $\operatorname{Spl}\left(T_{F}\right)=\left(t_{s}\right)_{s \in 2<n}$ be the set of splitting nodes of the tree $T_{F}$. By our assumption, it is $n$-increasing. For every $0 \leq j \leq n-1$, we let $L_{F}(j)=\left\{t_{s} \in\right.$ $\left.\operatorname{Spl}\left(T_{F}\right): s \in 2^{j}\right\}$. By the definition of $n$-increasing subtrees, the set $L_{F}(j)$ is the $j$-level of $\operatorname{Spl}\left(T_{F}\right)$ and so it is an antichain of $2^{<\omega}$. Let also $\operatorname{Spl}\left(T_{G}\right)$ be the set of splitting nodes of the tree $T_{G}$. Clearly $\operatorname{Spl}\left(T_{G}\right)$ is a subset of $\operatorname{Spl}\left(T_{F}\right)$.

Inductively, for every $0 \leq m \leq k-1$ we shall construct $j_{m} \in \omega$ and a subset $A_{m}$ of $2^{<\omega}$ such that the following are satisfied.

(1) $0 \leq j_{m} \leq n-1$ and if $m_{1}<m_{2}$, then $j_{m_{1}}>j_{m_{2}}$.

(2) $2^{j_{m}} \geq n^{k-m-1}$.

(3) $A_{m} \subseteq L_{F}\left(j_{m}\right)$ and $\left|A_{m}\right| \geq n^{k-m-1}$.

(4) If $0 \leq m_{1}<m_{2} \leq k-1$, then $A_{m_{2}}$ is a subset of $\operatorname{Spl}\left(\hat{A}_{m_{1}}\right)$.

(5) For all $0 \leq m \leq k-1$, we have $A_{m} \subseteq \operatorname{Spl}\left(T_{G}\right)$.

We start the construction. Notice that the family $\left\{\operatorname{Spl}\left(T_{G}\right) \cap L_{F}(j)\right\}_{j=0}^{n-1}$ forms a partition of $\operatorname{Spl}\left(T_{G}\right)$. Since $\left|\operatorname{Spl}\left(T_{G}\right)\right|=|G|-1 \geq n^{k}-1$ there exists $l \in\{0, \ldots, n-1\}$ such that $\left|\operatorname{Spl}\left(T_{G}\right) \cap L_{F}(l)\right| \geq n^{k-1}$. Notice that $\left|L_{F}(l)\right|=2^{l} \geq n^{k-1}$. We set $j_{0}=l$ and $A_{0}=\operatorname{Spl}\left(T_{G}\right) \cap L_{F}(l)$. Then conditions (2), (3) and (5) satisfied. This completes the first step of the inductive construction. As $A_{0}$ is an antichain, being a subset of $L_{F}\left(j_{0}\right)$, we have that $\left|\operatorname{Spl}\left(\hat{A}_{0}\right)\right|=\left|A_{0}\right|-1 \geq n^{k-1}-1$. As in the first step, we notice that the family $\left\{\operatorname{Spl}\left(\hat{A}_{0}\right) \cap L_{F}(j)\right\}_{j=0}^{j_{0}-1}$ forms a partition of $\operatorname{Spl}\left(\hat{A}_{0}\right)$. Hence, there exists $l^{\prime} \in\left\{0, \ldots, j_{0}-1\right\}$ such that $\left|\operatorname{Spl}\left(\hat{A}_{0}\right) \cap L_{F}\left(l^{\prime}\right)\right| \geq n^{k-2}$. We set $j_{1}=l^{\prime}$ and $A_{1}=\operatorname{Spl}\left(\hat{A}_{0}\right) \cap L_{F}\left(l^{\prime}\right)$. We proceed similarly.

We isolate the crucial properties established by the above construction.

(P1) For every $1 \leq m \leq k-1$ and every $w \in A_{m}$, the node $w$ has at least two successors in $A_{m-1}$.

(P2) For every $0 \leq m \leq k-1$, if $w_{1}, w_{2} \in A_{m}$ with $w_{1} \prec w_{2}$, then $\ell\left(w_{1}\right)<\ell\left(w_{2}\right)$.

(P3) For every $0 \leq m_{1}<m_{2} \leq k-1$, if $w_{1} \in A_{m_{1}}$ and $w_{2} \in A_{m_{2}}$, then $\ell\left(w_{1}\right)>\ell\left(w_{2}\right)$.

Property (P1) follows by (4) of the construction while properties (P2) and (P3) follow by (3) and (1) above and the fact that $\operatorname{Spl}\left(T_{F}\right)$ is $n$-increasing.

Using (P1)-(P3) and starting from a node in $A_{k-1}$ we construct a $k$-increasing subtree $T=\left(w_{s}\right)_{s \in 2<k}$ which is, by condition (5) of the inductive construction, a subset of $\operatorname{Spl}\left(T_{G}\right)$. This clearly implies the lemma. 
Lemma 14. Let $k \geq 1$ and $H \in[\mathcal{C}]_{\Delta}^{2^{k}}$. Then for every $(k+1)$-type $\tau$ there exists $I \subseteq H$ of type $\tau$.

Proof. By induction on $k$. If $k=1$, then the result is trivial since we can set $I=H$. Suppose that the result holds for some $k \geq 1$. Let $H \in[\mathcal{C}]_{\Delta}^{2^{k+1}}$ and $\tau:\{1, \ldots, k+1\} \rightarrow \omega$ be a $(k+2)$-type. Write $H$ in lexicographically increasing order as $H=\left\{y_{0}<\ldots<y_{2^{k+1}-1}\right\}$ and put $E=\left\{y_{i}: 0 \leq i<2^{k+1}, i\right.$ even $\}$. Let $\operatorname{Spl}\left(T_{H}\right)=\left(t_{s}\right)_{s \in 2^{<k+1}}$. It is easy to see that $\operatorname{Spl}\left(T_{E}\right)=\left(t_{s}\right)_{s \in 2<k}$ and so $E \in[\mathcal{C}]_{\Delta}^{2^{k}}$. Let $\tau^{\prime}=\left.\tau\right|_{\{1, \ldots, k\}}$. Then $\tau^{\prime}$ is a $(k+1)$-type. By our inductive assumption, there exists $I^{\prime} \subseteq E$ of type $\tau^{\prime}$. There exists $\left\{i_{0}<\ldots<i_{k}\right\} \subseteq\left\{0, \ldots, 2^{k}-1\right\}$ such that $I^{\prime}=\left\{y_{2 i_{0}}<\ldots<y_{2 i_{k}}\right\}$. We let $I=I^{\prime} \cup\left\{y_{2 i_{\tau(k+1)}+1}\right\}$. Then $I \subseteq G$ and it is easy to check that $I$ is of type $\tau$.

Lemma 15. Let $\mathcal{F} \subseteq[\mathcal{C}]^{<\omega}$ hereditary, $n \geq 2$ and $k \geq 2$ be such that $d_{\mathcal{F}}\left(2^{n}\right) \geq$ $n^{k-1}$. Then for every $P \subseteq \mathcal{C}$ perfect and every $k$-type $\tau$ there exists $I \in \mathcal{F} \cap[P]_{\tau}^{k}$.

Proof. As $P$ is perfect, there exists a $2^{n}$-increasing subset $F$ of $P$. Since $d_{\mathcal{F}}\left(2^{n}\right) \geq$ $n^{k-1}$, there exists $G \subseteq F$ with $G \in \mathcal{F}$ and $|G| \geq n^{k-1}$. Notice that $2^{n} \geq d_{\mathcal{F}}\left(2^{n}\right) \geq$ $n^{k-1}$. Hence, by Lemma 13, there exists $H \subseteq G$ which is $2^{k-1}$-increasing. By Lemma 14 there exists $I \subseteq H$ of type $\tau$. As $I \subseteq H \subseteq G \in \mathcal{F}$ and $\mathcal{F}$ is hereditary, the result follows.

We are ready to state and prove the main result of this section. To this end, we recall A. Blass' theorem [4] on partitions of $[\mathcal{C}]^{k}$, which states that if $U$ is open subset of $[\mathcal{C}]^{k}$ and $\tau$ is a $k$-type, then there exists $P \subseteq \mathcal{C}$ perfect (which is the body of a skew tree) such that either $[P]_{\tau}^{k} \subseteq U$ or $[P]_{\tau}^{k} \cap U=\varnothing$.

Theorem 16. Let $\mathcal{F} \subseteq[\mathcal{C}]^{<\omega}$ hereditary.

(1) Let $n \geq 2$ and $k \geq 1$ be such that $d_{\mathcal{F}}\left(2^{n}\right) \geq n^{k-1}$. If $\mathcal{F} \cap[\mathcal{C}]^{k}$ has the Baire property, then there exists $P \subseteq \mathcal{C}$ perfect such that $[P]^{k} \subseteq \mathcal{F}$.

(2) Assume that $\mathcal{F}$ has the Baire property in $[\mathcal{C}]^{<\omega}$ and satisfies

$$
(*) \quad \lim \sup \frac{\log _{2} d_{\mathcal{F}}\left(2^{n}\right)}{\log _{2} n}=+\infty \text {. }
$$

Then for every $k \geq 1$ there exists $P \subseteq \mathcal{C}$ perfect such that $[P]^{k} \subseteq \mathcal{F}$.

Proof. We argue first for part (1). If $k=1$ the result is trivial. So let $k \geq 2$ and assume that $\mathcal{F}$ has the Baire property in $[\mathcal{C}]^{k}$. By a classical result of J. Mycielski (see [7) and by passing to a perfect subset of $\mathcal{C}$, we may assume that $\mathcal{F} \cap[\mathcal{C}]^{k}$ is open. Fix a $k$-type $\tau$. By A. Blass' theorem there exists $P \subseteq \mathcal{C}$ perfect such that $[P]_{\tau}^{k}$ either is included in $\mathcal{F}$ or is disjoint from $\mathcal{F}$. The second alternative is impossible by Lemma 15. So the result follows by a finite exhaustion argument over all possible $k$-types. Part (2) follows from part (1) by a direct computation.

Remark 3. We do not know whether equation $(*)$ in Theorem 16(2) is the optimal one. We notice, however, that the conclusion of part (2) of Theorem 16 is not valid 
if we only assume that $\lim d_{\mathcal{F}}(n)=+\infty$. For instance, let $\mathcal{F}$ be the union of all strongly increasing and strongly decreasing finite subsets of $\mathcal{C}$ (recall that a subset $\left\{x_{0}<\ldots<x_{k}\right\}$ of $\mathcal{C}$ is said to be strongly increasing if $\ell\left(x_{i} \wedge x_{i+1}\right)<\ell\left(x_{i+1} \wedge x_{i+2}\right)$ for all $i \in\{0, \ldots, k-2\}$ - a strongly decreasing subset of $\mathcal{C}$ is similarly defined). Then $\mathcal{F}$ is closed in $[\mathcal{C}]^{<\omega}$ and it is easy to verify that $\lim d_{\mathcal{F}}(n)=+\infty$. However, for every $k \geq 4$ there does not exist a perfect subset $P$ of $\mathcal{C}$ with $[P]^{k} \subseteq \mathcal{F}$.

Consequences. We start with the following proposition which shows that the families presented in Example 1 are essentially the only ones within $C$-measurable hereditary families which satisfy $(*)$.

Proposition 17. Let $\mathcal{F} \subseteq[\mathcal{C}]^{<\omega}$ hereditary. Assume that $\mathcal{F}$ is $C$-measurable in $[\mathcal{C}]^{<\omega}$ and satisfies equation $(*)$ of Theorem 16 . Then for every $g: \omega \rightarrow \omega$ and every $P \subseteq \mathcal{C}$ perfect there exists a regular dyadic subtree $T=\left(t_{s}\right)_{s \in 2}<\omega$ with $[\hat{T}] \subseteq P$ and such that $\mathcal{G} \subseteq \mathcal{F}$, where $\mathcal{G}=\bigcup_{k \in \omega} \bigcup_{s \in 2^{k}}\left\{G: G \subseteq[\hat{T}]_{t_{s}}\right.$ and $\left.|G| \leq g(k)\right\}$.

Proof. By Theorem 16 and our assumptions, we have that for every $P \subseteq \mathcal{C}$ perfect and every $m \geq 1$ there exists $Q \subseteq P$ perfect with $[Q]^{m} \subseteq \mathcal{F}$. Hence, arguing as in Lemma 4. we may construct a regular dyadic subtree $T=\left(t_{s}\right)_{s \in 2}<\omega$ and a family $\left(P^{s}\right)_{s \in 2^{<\omega}}$ of perfect subsets of $P$ such that $t_{\varnothing}=\varnothing$ and moreover the following hold.

(i) For every $k \in \omega$, every $s \in 2^{k}$ and every $i \in\{0,1\}, P^{s^{\wedge}} \subseteq P^{s} \cap \mathcal{C}_{t_{s}>i}$.

(ii) For every $k \in \omega$ and every $s \in 2^{k},\left[P^{s}\right]^{g(k)} \subseteq \mathcal{F}$.

Clearly $T$ is as desired.

We need to introduce some more terminology. Let $f: \omega \rightarrow \omega$ be such that $n \geq f(n)>0$ for all $n \geq 1$. Let also $\mathcal{F} \subseteq[\mathcal{C}]^{<\omega}$ and $A \subseteq \mathcal{C}$. We say that $\mathcal{F}$ is $f$-filling over $A$ if for every $n \geq 1$ and every $F \subseteq A$ with $|F|=n$ there exists $G \subseteq F$ with $G \in \mathcal{F}$ and $|G| \geq f(n)$. We notice that if $\mathcal{F} \subseteq[\mathcal{C}]^{<\omega}$ is an arbitrary hereditary family with $\lim d_{\mathcal{F}}(n)=+\infty$, then for every $A \subseteq \mathcal{C}$ infinite there exists $B \subseteq A$ countable such that $\mathcal{F}$ becomes $1 / 2$-filling over $B$ (this follows by an application of Ramsey's theorem). Although, by Theorem 2 this fact cannot be extended to perfect sets, it can be extended for weaker versions of density as the following corollary demonstrates.

Corollary 18. Let $\mathcal{F} \subseteq[\mathcal{C}]^{<\omega}$ be as in Proposition 17 and $f: \omega \rightarrow \omega$ be such that $n \geq f(n)>0$ for all $n \geq 1$ and $\lim \frac{f(n)}{n}=0$. Then for every $P \subseteq \mathcal{C}$ perfect there exists $Q \subseteq P$ perfect such that $\mathcal{F}$ is $f$-filling over $Q$.

Proof. We may select a strictly increasing sequence $\left(n_{k}\right)_{k \in \omega}$ such that $n_{0}=1$ and $\sup _{i \geq n_{k}} \frac{f(i)}{i} \leq \frac{1}{2^{k}}$ for all $k \in \omega$. We define $g: \omega \rightarrow \omega$ by $g(k)=\left\lceil n_{k+1} / 2^{k}\right\rceil$. Let $T=\left(t_{s}\right)_{s \in 2<\omega}$ be the regular dyadic subtree obtained by Proposition 17 for the function $g$ and the given perfect set $P$. Setting $Q=[\hat{T}]$ and arguing as in Example 1, we can easily verify that $Q$ has all the desired properties. 


\section{Connections With Banach spaCes}

Theorem 2 has some Banach space theoretic implications which we are about to describe. Let $\mathcal{F} \subseteq[\mathcal{C}]^{<\omega}$ hereditary with $[\mathcal{C}]^{1} \subseteq \mathcal{F}$. For every such family $\mathcal{F}$ we define a Banach space $X_{\mathcal{F}}$ as follows. Let $c_{00}(\mathcal{C})$ be the vector space of all real-valued functions on $\mathcal{C}$ with finite support and denote by $\left(e_{x}\right)_{x \in \mathcal{C}}$ the standard Hamel basis of $c_{00}(\mathcal{C})$. Then $X_{\mathcal{F}}$ is the completion of $c_{00}(\mathcal{C})$ under the norm $\|\cdot\|_{\mathcal{F}}$ defined by

$$
\left\|\sum_{i=0}^{n} a_{i} e_{x_{i}}\right\|_{\mathcal{F}}=\sup \left\{\sum_{i \in F}\left|a_{i}\right|:\left\{x_{i}: i \in F\right\} \in \mathcal{F}\right\} .
$$

We recall that a bounded sequence $\left(e_{n}\right)_{n}$ in a Banach space $E$ is called Cesaro summable if the sequence of averages $\frac{e_{0}+\ldots+e_{n-1}}{n}$ converges in norm. Under the above terminology we have the following proposition.

Proposition 19. Let $\mathcal{F} \subseteq[\mathcal{C}]^{<\omega}$ be hereditary, compact and such that $[\mathcal{C}]^{1} \subseteq \mathcal{F}$. Assume that $\mathcal{F}$ is $C$-measurable and $\lim d_{\mathcal{F}}(n)=+\infty$. Then the following hold.

(1) For every sequence $\left(x_{i}\right)_{i}$ in $\mathcal{C}$ there exists $L \subseteq \omega$ infinite such that for every $N \subseteq L$ infinite the sequence $\left(e_{x_{i}}\right)_{i \in N}$ is not Cesaro summable in $X_{\mathcal{F}}$.

But on the other hand,

(2) for every $P \subseteq \mathcal{C}$ perfect, there exists $\left(x_{i}\right)_{i}$ in $P$ such that the sequence $\left(e_{x_{i}}\right)_{i}$ is Cesaro summable in $X_{\mathcal{F}}$.

Proof. (1) Let $\left(x_{i}\right)_{i}$ be a sequence in $\mathcal{C}$. As we have already remarked, by the fact that $\mathcal{F}$ is hereditary and $\lim d_{\mathcal{F}}(n)=+\infty$, there exists $L \subseteq \omega$ infinite such that $\mathcal{F}$ is $1 / 2$-filling over $\left\{x_{i}: i \in L\right\}$. By the definition of the norm of $X_{\mathcal{F}}$, we see that for every $F \subseteq L$ finite we have $\left\|\sum_{i \in F} e_{x_{i}}\right\|_{\mathcal{F}} \geq \frac{|F|}{2}$. This clearly implies that for every $N \subseteq L$ infinite the sequence $\left(e_{x_{i}}\right)_{i \in N}$ is not Cesaro summable in $X_{\mathcal{F}}$.

(2) Let $P \subseteq \mathcal{C}$ perfect. By our assumptions, Lemma 4 can be applied. Hence, there exists a regular dyadic subtree $T=\left(t_{s}\right)_{s \in 2<\omega}$ that decides for $\mathcal{F}$ and $[\hat{T}] \subseteq P$.

Let $Z$ be the set of all eventually zero sequences in $\mathcal{C}$. We enumerate $Z$ as $\left(z_{i}\right)_{i \in \omega}$ as follows. For every $i \in \omega$ let $z_{i}$ be the unique element of $Z$ satisfying $i=\sum_{k \in \omega} z_{i}(k) 2^{k}$. By the uniqueness of the dyadic representation of every natural number, we have that if $i \neq j$, then $z_{i} \neq z_{j}$ and moreover, if $n, i, j \in \omega$ are such that $i, j<2^{n}$, then $z_{i}\left|n \neq z_{j}\right| n$.

For every $i \in \omega$ define $x_{i}=\bigcup_{k \in \omega} t_{z_{i} \mid k} \in[\hat{T}]$. We claim that $\left(x_{i}\right)_{i \in \omega}$ is the desired sequence. To this end, for every $n \in \omega$ let $s \in 2^{n}$ and put $l_{n}=\ell\left(t_{s}\right.$ ) (as $T$ is regular dyadic $l_{n}$ is well-defined and independent of the choice of $s$ ). By the above mentioned property of the sequence $\left(z_{i}\right)_{i}$, for every $i, n \in \omega$ with $i<2^{n}$ we have that

$$
\left|\left\{x_{0}\left|l_{n}, \ldots, x_{i}\right| l_{n}\right\}\right|=\left|\left\{x_{0}, \ldots, x_{i}\right\}\right|=i+1
$$


For every $n \in \omega$ define

$$
M_{n}=\max \left\{|F|: F \subseteq 2^{n} \text { and } F \text { is trapped in } \mathcal{F}\right\} .
$$

By (11) and the fact that the tree $T$ decides for $\mathcal{F}$, for every $i<2^{n}$ we get that

$$
\max \left\{|G|: G \subseteq\left\{x_{0}, \ldots, x_{i}\right\} \text { and } G \in \mathcal{F}\right\} \leq M_{n} .
$$

Let $i, n \geq 1$ with $2^{n-1} \leq i<2^{n}$. Then

$$
\left\|\frac{1}{i+1} \sum_{k=0}^{i} e_{x_{k}}\right\|_{\mathcal{F}} \leq \frac{M_{n}}{i+1} \leq \frac{M_{n}}{2^{n-1}}=2 \frac{M_{n}}{2^{n}} .
$$

Finally notice that

$$
\lim \frac{M_{n}}{2^{n}}=0 .
$$

For if not, arguing as in the proof of Lemma 6, we would have that there exists $R \subseteq[\hat{T}]$ perfect with $[R]^{<\omega} \subseteq \mathcal{F}$, contradicting the fact that $\mathcal{F}$ is compact. Hence, by (2), we have

$$
\frac{1}{i+1} \sum_{k=0}^{i} e_{x_{k}} \rightarrow 0
$$

and the proof is completed.

Remark 4. (a) Part (2) of Proposition 19 can also be derived by Theorem 3A in 6], taking into account that every $C$-measurable, hereditary and compact family $\mathcal{F}$ is not $\varepsilon$-filling for every $\varepsilon>0$. For completeness we have included a proof in the present setting.

(b) We notice that the fact that every subsequence of the sequence $\left(x_{i}\right)_{i \in L}$, obtained in part (1) of Proposition 19, is not Cesaro summable, is expected by the ErdösMagidor theorem [5] (see also [2]).

(c) We notice that under the assumptions of Proposition 19, for every $P \subseteq \mathcal{C}$ perfect there exists $Q \subseteq P$ perfect with the following property. If $\left(x_{i}\right)_{i}$ is a sequence in $Q$ and the sequence $\left(e_{x_{i}}\right)_{i}$ generates a spreading model (see [2] for the definition), then this spreading model must be $\ell_{1}$.

\section{REFERENCES}

[1] A. W. Apter and M. Džamonja, Some remarks on a question of D. H. Fremlin regarding E-density, Arch. Math. Logic, 40 (2001), 531-540.

[2] S. A. Argyros and S. Todorčević, Ramsey Methods in Analysis, Advanced Courses in Mathematics, CRM Barcelona, Birkhäuser Verlag, Basel, 2005.

[3] H. Becker and A. S. Kechris, The Descriptive Set Theory of Polish Group Actions, LMS Lecture Note Series, 232 (1996), Cambridge Univ. Press.

[4] A. Blass, A partition theorem for perfect sets, Proc. AMS, 82 (1981), 271-277.

[5] P. Erdös and M. Magidor, A note on regular methods of summability and the Banach-Saks property, Proc. AMS, 59 (1976), 232-234.

[6] D. H. Fremlin, Problem DU, available at www.essex.ac.uk/maths/staff/fremlin/problems.htm.

[7] A. S. Kechris, Classical Descriptive Set Theory, Grad. Texts in Math., 156, Springer-Verlag, 1995. 
[8] A. Louveau, S. Shelah and B. Veličković, Borel partitions of infinite subtrees of a perfect tree, Annals Pure Appl. Logic, 63 (1993), 271-281.

[9] R. M. Solovay, A model of set theory in which every set of reals is Lebesgue measurable, Annals of Math., 92 (1970), 1-56.

National Technical University of Athens, Faculty of Applied Sciences, Department of Mathematics, Zografou Campus, 157 80, Athens, Greece

E-mail address: pdodos@math.ntua.gr, bkanel@math.ntua.gr 